\title{
Views of South African biomedical research ethics committee members on their own ethics review outcomes
}

\author{
B Silaigwana, MSc, PhD; D Wassenaar, MA Clin Psych, PhD \\ School of Applied Human Sciences, College of Humanities, University of KwaZulu-Natal, Pietermaritzburg, South Africa
}

Corresponding author: B Silaigwana (silaigwana@yahoo.com)

\begin{abstract}
Background. Current research ethics guidelines and legislation provide research ethics committees (RECs) with the prerogative to review and approve the ethical acceptability of human research before commencement. However, individual REC members' views on the ethical issues identified behind closed doors remain largely empirically unexplored.

Objective. To investigate the views and perceptions of South African biomedical REC members on their own aggregated ethics review outcomes. Methods. Semi-structured interviews were conducted with nine REC members from two different institutions in South Africa.

Results. All respondents concurred with the prioritisation of informed consent in the review outcomes, emphasising the importance of the use of simple and understandable language for participants. Respondents also discussed lack of scientific validity as a common problem when reviewing proposals. Interestingly, while the majority of respondents reiterated the common view that scientifically invalid research is unethical, some REC members believed that there was an overemphasis on evaluation of scientific validity, and that it was not within the remit of RECs to review the scientific quality of proposed studies. The REC members felt that HIV and tuberculosis research had high social value, because it addresses national and regional public health priorities. For this reason, there was no concern expressed that a high proportion of research proposals involved HIV and TB.

Conclusion. Although most respondents found the aggregated results unsurprising, there was some disagreement with the ranked review outcomes, with a minority of respondents arguing that scientific validity was overemphasised. These findings have potential training and practice implications for RECs and researchers. The study findings call for further studies to validate such findings in other RECs.
\end{abstract}

SAfr J Bioethics Law 2019;12(1):8-13. DOI: 10.7196/SAJBL.2019.v12i1.654

Research ethics committees (RECs; known as institutional review boards (IRBs) in the USA) are an integral part of the human research participants' protection system. ${ }^{[1]}$ They have the authority to approve, conditionally approve or reject research proposals, depending on the committee's determination of a study's ethical acceptability, as judged in terms of local and international guidance..$^{[1]}$ Unfortunately, RECs generally operate behind closed doors, meaning that there is little understanding of REC reviews in practice. Two books, Behind Closed Doors: IRBs and the Making of Ethical Research ${ }^{[2]}$ and Ethics Police: The Struggle to Make Human Research Safe, ${ }_{1}^{[3]}$ provide important insights into the work of IRBs in the USA. However, research into the operation of RECs is still relatively limited in developing countries such as South Africa (SA).

While RECs play an important role in ensuring the protection of research participants, they are often heavily criticised for overstepping their scope, being too bureaucratic, delaying important research and spending too much time rewording informed consent forms. ${ }^{[3,4]}$ Some social science commentators have even called for the abolition of REC reviews, arguing that mandatory ethics review is itself unethical because RECs do not respect researchers or each other, lack merit and integrity and are neither just nor beneficent. ${ }^{[5]}$

Despite such criticism, there have been relatively few empirical studies exploring the work of RECs, although some studies ${ }^{[6-9]}$ have examined REC minutes and decision letters retrospectively to identify the ethical issues raised, both internationally and in SA. These studies have suggested that RECs frequently raise ethical issues related to informed consent, scientific validity and respect for persons, while issues such as risk/benefit seem to receive less attention. However, empirical studies exploring individual REC members' views and perspectives on the ethical issues identified during ethics reviews are surprisingly scarce, despite the potential utility of such data in informing future training for REC members on how the committees should assess and/or prioritise these issues. ${ }^{[3]}$ In a related study, we reported data on aggregated ranked ethical issues raised by the two SA biomedical RECs included in this study, and found that these issues were ranked in the following descending order of frequency: informed consent; respect for participants; scientific validity; administrative queries; collaborative partnership; editorial errors; fair participant selection; favourable risk/benefit ratio; independent ethics review; and social value. ${ }^{[9]}$ The present paper explores REC members' perceptions of and comments on those findings, to see if they align with their own perceptions of what RECs should be highlighting in the ethics review process. We were therefore interested in gaining a better understanding of the views of individual REC members on the aggregated ranked ethical issues from the two RECs combined. ${ }^{\left[{ }^{[9]}\right.}$ Such data are relevant because there is no agreed normative framework for interpreting the ranking or optimal frequency of types of ethical queries. For this reason, we conducted qualitative interviews with REC members to explore their perceptions of the ranked distribution of the ethical issues in the earlier study. ${ }^{[9]}$ 


\section{Methods}

The present study was approved by RECs at both research sites (no names or ethics clearance reference numbers can be provided, to preserve confidentiality under the conditions of non-disclosure agreements). Gatekeeper permissions were also obtained from the relevant institutional authorities. All the interviewees gave voluntary informed consent. The two participating RECs, and the REC members, were purposively selected based on availability and willingness to participate, without any bias or unfair predetermined inclusion/ exclusion criteria. The potential risk of harm to the reputation of either of the RECs or their affiliated institutions has been offset by anonymisation and the strict maintaining of confidentiality.

\section{Design and rationale}

Semi-structured interviews allow an in-depth understanding of how individuals experience or understand a particular phenomenon in their context. Unlike quantitative methods that focus on breadth, representativeness and generalisability, semi-structured interviews do not necessarily aim to have a representative sample, but rather focus on the depth, insight and transferability of findings. ${ }^{[10]}$ Therefore, given that the aim of the study was to explore the views of REC members regarding ethical issues identified during ethics review, we believed the best method to be semi-structured interviews.

\section{Participant sampling strategy}

Given that RECs are notoriously difficult to gain access to for research purposes, ${ }^{[11]}$ the first author initially approached the REC chairs at each respective site to request assistance with the recruitment of REC members. Various attempts were made to recruit members from each REC, but most were met with non-response or refusal. This was not unexpected, as several reports have suggested that RECs are generally reluctant to be researched, suggesting that more needs to be done to encourage them to accept that their work is a legitimate research focus. ${ }^{[9,11]}$

In REC 1, the chair (after informing REC members about the study during a monthly meeting) granted the first author access to the names and contact details of all REC members. Thereafter, a recruitment email was sent to each member, inviting them to volunteer to participate. Four of 22 members responded to this email and expressed willingness to participate. Follow-up reminder emails were sent to the remaining REC members who had not responded. Another 3 members responded, but 2 withdrew, citing time constraints. A third and final follow-up email was sent to remaining members who had not previously responded. There was no further response, and so a total of 5 members from REC 1 consented to participate in the study.

In REC 2, the chair recommended that a group email be sent to all members, inviting them to participate in the study. Two members consented to participate. After a month, reminder emails were sent to those members who had not initially responded. A further 2 responded and consented to participate. A third and final reminder email was sent to members who had not responded to the previous emails, but there was no further response. A total of 4 members from REC 2 agreed to participate in the study.

Overall, 9 members from the 2 RECs consented to take part in this study. Each member was then provided with graphs showing the aggregated frequency rankings of the ethical issues that had been raised by both their RECs combined. ${ }^{[9]}$ Semi-structured interviews 45 60 minutes long were conducted face-to-face or by telephone, using an interview guide. Probes, requests for clarification and follow-up questions were used to elicit respondents' views and impressions of the issues revealed. The interviews were recorded, and transcribed verbatim.

\section{Data analysis}

Thematic analysis was used to code and analyse the narrative data from the transcripts and to identify salient themes emerging from the data. This method facilitates a consistent, systematic and replicable means of examination of data. ${ }^{[10]}$ Data were analysed both inductively and deductively to identify the themes, using a pre-existing ethics framework. ${ }^{[12]}$ Emerging codes and themes were sought by disaggregating the data and constantly comparing them for similarities and differences.

\section{Results}

\section{Response rate}

In total, 66 REC members were invited to participate. Of the 2 groups, 5 members of REC 1 and 4 of REC 2 consented to participate. Overall, there was a $14 \%(9 / 66)$ response rate. The sample was slightly skewed in favour of REC 1 , despite efforts to increase enrolment from REC 2.

\section{Demographic characteristics}

Table 1 summarises respondents' demographic characteristics. The majority (77.7\%) were white academics employed by the institutions to which each REC was affiliated. All but one had a PhD in a health science field, with almost equal numbers of members from medicine, public health and biomedical sciences. No lay members volunteered to take part in the study. In terms of gender, there was a 4:5 male:female ratio of respondents. Their length of experience as REC members was similar between the groups, ranging from 2 - 10 years.

\section{Thematic analysis}

We report on the main themes emerging from the qualitative data. The first question asked of respondents was what they thought the main role of research ethics review was. All respondents felt that their primary task was to protect research participants and provide adequate ethics oversight for research:

'I think RECs primarily ensure participant protection, and I see it particularly from a medical point of view, where there has been a history of abuse of research participants in medical science ... And then there are other reasons that come about, to make sure that the research is of good quality, that the researchers actually know what they are doing when it comes to doing research ... so we have ethics now because we don't want participants to ever be abused again!

However, another respondent added that while RECs are primarily there to protect participants, they should not stifle research:

My way of looking at the ethics committee is that it's there to protect participants, but at the same time not stifle research which is where I end up conflicting often with some of my colleagues who seem to suggest that we [RECs] should be stifling research ... But, so I like to look at it from, is this study in general going to be safe, doable and practicable without putting [a] patient or participant's safety at risk?' 
Table 1. Demographic characteristics of respondents

\begin{tabular}{|c|c|c|c|}
\hline Characteristic & REC 1, $n(\%)$ & $\operatorname{REC} 2, n(\%)$ & Total, $n(\%)$ \\
\hline \multicolumn{4}{|l|}{ Race } \\
\hline Black African & $1(20)$ & $0(0)$ & $1(11)$ \\
\hline Indian & $1(20)$ & $0(0)$ & $1(11)$ \\
\hline White & $3(60)$ & $4(100)$ & $7(78)$ \\
\hline \multicolumn{4}{|l|}{ Gender } \\
\hline Female & $2(40)$ & $3(75)$ & $5(56)$ \\
\hline Male & $3(60)$ & $1(25)$ & $4(44)$ \\
\hline \multicolumn{4}{|l|}{ Age group, years } \\
\hline $30-40$ & $0(0)$ & $3(75)$ & $3(33)$ \\
\hline $41-50$ & $1(20)$ & $1(25)$ & $2(22)$ \\
\hline $51-60$ & $4(80)$ & $0(0)$ & $4(44)$ \\
\hline$>61$ & $0(0)$ & $0(0)$ & $0(0)$ \\
\hline \multicolumn{4}{|l|}{ Highest education level } \\
\hline $\mathrm{PhD}$ & $5(100)$ & $3(75)$ & $8(89)$ \\
\hline Masters & $0(0)$ & $1(25)$ & $1(11)$ \\
\hline \multicolumn{4}{|l|}{ Area of specialisation } \\
\hline Medicine & $3(60)$ & $2(50)$ & $5(56)$ \\
\hline Public health & $1(20)$ & $1(25)$ & $2(22)$ \\
\hline Psychology & $0(0)$ & $1(25)$ & $1(11)$ \\
\hline Biomedical science & $1(20)$ & $0(0)$ & $1(11)$ \\
\hline \multicolumn{4}{|l|}{ Role in REC } \\
\hline Chair/co-chair & $1(20)$ & $2(50)$ & $3(33)$ \\
\hline Internal member & $4(80)$ & $2(50)$ & $6(67)$ \\
\hline Lay member & $0(0)$ & $0(0)$ & $0(0)$ \\
\hline Experience as REC member, range (years) & $(2-6)$ & $(2-10)$ & \\
\hline
\end{tabular}

In the next set of questions, respondents were asked to describe the kinds of ethical issues that they typically identify during an ethics review, and their opinions on the relative frequency of these issues, presented below in descending order.

\section{Informed consent}

Although the sample was relatively small $(n=9)$, all respondents identified informed consent as the issue that they most commonly find problematic in research protocols:

'It's almost common sense to think that if informed consent is not in place that's probably not going to be ethical research ... without it you can automatically say that this study is unlikely to be ethical, unless of course if there are other circumstances that allow for waivers of informed consent. So, for me [informed consent] is the first step in actually making sure that research is ethical, and the [other ethical issues] follow.'

When asked to elaborate further on the key elements of informed consent that they identified as problematic, almost all respondents mentioned that the most frequent problems include the use of too much technical language on consent forms, and/or failing to provide sufficient detail about the study procedures. Therefore, REC members stated that they often required changes to the informed consent document in terms of simplification of language:

'We tend to focus on informed consent; it has to be thorough. Usually we receive a huge document, and the researcher says this is informed consent, and we say "No, no, no! Simplify it, simplify the language, it's too technical." It doesn't matter whether the funders wanted it that way, but we want it to be simple for the participants, so the language must be simplified and it must be shortened and list everything that is there, including the harms.'

\section{Respect for participants}

Regarding the principle of respect for participants, most REC members said that they are often troubled when there is no clear indication from researchers and their sponsors on plans for ongoing medical care for participants after the study has been completed.

'One of the common issues in terms of participant protection is the issue of post-trial availability of test drugs, okay. And that's something I stare [at] in horror with some of the commercial proposals that get sent to me for review.

\section{Scientific validity}

While all REC members acknowledged the importance of scientific validity for any research, there were divergent views regarding the frequency of scientific queries raised by RECs in this study. Most (7/9) respondents said they were not surprised by the high frequency of scientific queries:

'Scientific validity is at the roots of research and immediately if the scientific validity is wrong, is misinterpretable, then... there is no point looking at the ethics. If it's not scientifically valid, it's immediately unethical to start the study.'

Interestingly, however, two respondents strongly believed there was often an over-emphasis on scientific issues that fall outside the remit of RECs: 
'One of the biggest issues that strikes me the most ... is the overemphasis on the scientific validity ... We tend to lose focus that we are ethics committees reviewing ethical issues, noting that the science should be ethical. But we are not there to ask all these questions about the scientific methodology.'

\section{Collaborative partnerships}

REC members were concerned about the need for collaborative partnerships with local researchers, and respect for local culture:

'There are other studies that are internationally funded, and [have] bigger sponsors. So essentially the sponsor pushes through what is applicable in their settings, and these are from developed countries, and they push through the norms and standards they use and they expect that to be done here also, with little regard to the local issues and the cultural sensitivities and the local guidelines that apply here.'

\section{Social value}

When asked about social value, all respondents generally felt that because most proposals reviewed by their RECs addressed health issues of national importance, such as HIV and tuberculosis (TB), they had high social value:

'The nature [of the studies reviewed by the REC] is biomedical research, and they are public health research. So when the researchers come, they study those issues that are of national priority, so they justify why they want to do their research. Like I said, HIV and TB is very topical in SA ... So obviously once you [the REC member] look at [the proposal], the reviewer will know that this is a national priority and they will say okay there is social value because SA is battling with HIV, you know ... the issues are easy to sort.

\section{Administrative issues}

Furthermore, REC members said that they often raised administrative queries, such as missing investigator curriculum vitae (CVs) or research budgets, because although these are not overtly ethical issues, they do have ethical and scientific implications. To illustrate this point, one respondent said:

'For instance, with CVs and qualifications, it comes back to ... are the researchers qualified or experienced in what they are researching, okay? You don't want to send out a boy to do a man's job [laughter] because it's not fair on the participants; you can't send somebody who doesn't know what they are doing to do research on these people.'

Importantly, most REC members noted that most of these administrative issues, such as CVs, are in fact regulatory requirements in accordance with national guidance:

'Quite a lot of these things [documents] are regulatory requirements. The NHREC [National Health Research Ethics Council] regulations require that these things [documents ] are included in ethics review applications - and if they are not there they invalidate the application, and it means the REC is doing something wrong by keeping on file an application that is incomplete without those things.'.

In summary, the respondents believed that they did not necessarily over-scrutinise protocols, but raised issues simply because they had not been properly addressed, or were missing from the submitted proposals. As such, REC members could not simply turn a blind eye to those issues. One respondent said:

'I don't think there is any terrible plot from RECs to pick everybody up on these issues. It's just that they are there; if it's an issue it's an issue. You can't just say, oh well, I'm going to ignore this because I have been sending a lot of these queries back recently. You can't say it's wrong to pick up on it, you can't say a lot of these are going back [to applicants], let's try and decrease our numbers [of queries] ... So this reflects us doing our job - it's not a policing thing.'

\section{Discussion}

There have been many calls for evaluation of RECs, ${ }^{[13]}$ despite an ongoing lack of consensus on the assessment criteria for evaluating research ethics reviews. ${ }^{[14]}$ The present article reports on SA REC members'views regarding ranked aggregated ethical issues identified in their review work. Most importantly, all REC members mentioned informed consent as the cornerstone for ethical research. The results in our study are comparable to findings reported in similar studies elsewhere. For instance, a study in the USA found that IRB chairs often cited concerns with the length, complexity and reading level of informed consent forms. ${ }^{[15]}$ However, another study investigating how US IRBs make decisions about consent forms found that while IRBs generally strive to decrease the length and complexity of consent forms, some IRB members often disagreed on what and how much detail the forms should include. ${ }^{[16]}$

We found divergent views regarding the frequency of REC queries on scientific validity. As highlighted in the results, some respondents expressed concern about an overemphasis on scientific issues; they said they believed that querying the science falls outside REC scope. While national ${ }^{[17]}$ and international ${ }^{[18]}$ ethical guidelines clearly state that REC approval should require proof of sufficient scientific validity to answer the primary research question, it remains unclear what researchers themselves think about the role of RECs in reviewing the scientific quality of their proposals. Klitzman ${ }^{[19]}$ found that IRB members often encounter dilemmas about whether proposals should have a separate science review, and how good the science needs to be for the study to be valid.

REC members highlighted the need for meaningful community engagement in international research, echoing long-standing ethical concerns about exploitation when research is sponsored by wealthy countries and conducted in less-developed countries. ${ }^{[20]}$ Empirical studies have reported that local RECs and other stakeholders frequently express concerns about developing partnerships, building sustainable local scientific capacity and benefit-sharing. ${ }^{[21]}$ Several commentators acknowledge social value as an important ethical benchmark, in that a study should contribute to scientific knowledge generation and improve healthcare for both current and future patients. ${ }^{[12,22,23]}$ There are few empirical studies that have explored stakeholders' views on social value in research. A study in Kenya ${ }^{[24]}$ found that stakeholders considered a study to have social value if it involved benefit-sharing, for example, through post-trial access to medical care, and societal benefits emanating from the successful completion of research.

The present study also found that REC members frequently raised administrative queries, e.g. missing investigator CVs and editorial errors. This suggests that researchers definitely need to proofread their ethics applications, and check that they have included all the 
information and documentation required, before submission. ${ }^{[25]} \mathrm{A}$ UK study similarly found that RECs frequently identify editorial errors and missing documents in applications for ethics approval. ${ }^{[26]}$ It could be argued that editorial and administrative issues reflect poor attention to detail by researchers, which could be viewed by RECs as a predictor of similar poor oversight in the study being proposed. Alternatively, or in addition, RECs could be briefed to attend only to editorial issues if they obscure key meanings of ethical import. Further work is needed to articulate and refine RECs' concerns arising under this general additional category.

We were somewhat surprised that respondents were satisfied with the relatively low ranking of reported queries about favourable risk/ benefit ratio and, until data show anything to the contrary, can only assume that such issues are generally better dealt with in the ethics protocols than the ethical issues discussed above.

These findings have educational implications for both RECs and researchers. Both stakeholder groups should continually aim to understand the key ethical issues applicable to human participant research, and learn how to better address such issues in applications for ethics approval. There is a real opportunity for RECs to be more open with researchers, and advise them amicably in advance of protocol submission to flag and address potential ethical issues, ${ }^{[25]}$ in order to avoid the perception that they are 'ethics police. ${ }^{\text {[3] }}$

There are potential limitations to the present study. First, we only interviewed 9 REC members, from 2 participating SA RECs. ${ }^{\left[{ }^{[9]}\right.}$ Hence the findings reported here might not reflect the views of other REC members from the 44 RECs registered with the SA National Health Research Ethics Council. Future studies could do qualitative interviews with larger samples consisting of all SA RECs. Further quantitative research could also be done to assess REC members' and researchers' opinions on the optimal $v$. reported ranking of ethical issues arising from ethics review. Another limitation is that the respondents knew that they were, partly, indirectly reviewing their own REC's work, which could have inclined them to be uncritical. A future study should seek commentary from independent REC members or other research ethics experts on the same aggregated data set. It is also possible that the quality of data from the qualitative interviews was influenced by the interviewer's qualitative interviewing skills. Finally, we did not interview researchers who submitted proposals to the RECs sampled in this study; future studies might be needed to investigate how researchers view and perceive the ethical issues raised by their RECs.

\section{Conclusion}

The qualitative data presented in this article summarise the views of 9 SA REC members on their own RECs' reviews. In agreement with the ranked data, REC members generally considered informed consent to be the most important ethical issue arising during ethics review. However, there were some disagreements among REC members on the extent to which RECs should concern themselves with issues of scientific validity. While there is no agreed-upon normative framework to interpret the ranking or frequency of ethical queries raised by RECs, qualitative interviews with REC members in this study confirmed that the distribution of the ethical issues raised was roughly what they would expect during ethics review. The data presented here potentially contribute to a better understanding of REC review, as it generally occurs behind closed doors. ${ }^{[2,11]}$ Areas of further study are suggested above, to expand on and explore such findings in other research ethics settings.

Acknowledgements. We are most grateful to the management and administrative staff of both participating universities for giving us access to and trusting us with their REC/IRB meeting minutes.

Author contributions. BS was responsible for data collection, analysis, preparation of the first draft and approval of the final version. DW contributed in various ways to the work reported in this article, including guidance in data analysis, writing and approval of final version.

Funding. This article was partially supported by grant no. 5R24TW008863 from the President's Emergency Plan for AIDS Relief (PEPFAR), and the National Institutes of Health, US Department of Health and Human Services, through the MEPI award. Its contents are solely the responsibility of the authors, and do not necessarily represent the official views of the US government.

\section{Conflicts of interest. None.}

1. Department of Health and Human Services, USA. International compilation of human research standards. Washington, DC: DHHS, 2018. https://www.hhs.gov/ ohrp/sites/default/files/2018-International-Compilation-of-Human-ResearchStandards.pdf (accessed 10 June 2018).

2. Stark L. Behind Closed Doors: IRBs and the Making of Ethical Research. Chicago, IL: Chicago University Press, 2012

3. Klitzman R. The Ethics Police? The Struggle to Make Human Research Safe. New York: Oxford University Press, 2015.

4. Abbott L, Grady C. A systematic review of the empirical literature evaluating IRBs: What we know and what we still need to learn. J Empir Res Hum Res Ethics 2011;9(5):36-45. https://doi.org/10.1525/jer.2011.6.1.3

5. Dyck $M$, Allen $G$. Is mandatory research ethics reviewing ethical? J Med Ethics 2012;39(1):517-520. https://doi.org/10.1136/medethics-2011-100274

6. Cleaton-Jones P. Process error rates in general research applications to the human research ethics committee (medical) at the University of the Witwatersrand: A secondary data analysis. S Afr J Bioethics Law 2010;3(1):2024.

7. Clarke DL. Auditing the process of ethics approval for master's degrees at a South African university. S Afr J Bioethics Law 2014;7(1):23-25. https://doi.org/10.7196/ sajbl.301

8. Tsoka-Gwegweni J, Wassenaar DR. Using the Emanuel et al. framework to examine ethical issues raised by a biomedical research ethics committee in South Africa. J Empir Res Hum Res Ethics 2014;9(5):36-45. https://doi. org/10.1177/1556264614553172

9. Silaigwana B, Wassenaar D. Research ethics committees' oversight of biomedical research in South Africa: A thematic analysis of ethical issues raised during ethics review of non-expedited protocols. J Empir Res Hum Res Ethics 2019;14(2):107-116.

10. Patton MQ. Qualitative Research and Evaluation Methods. 3rd edition. New York: Sage Publications, 2002.

11. Fernandez-Lynch H. Opening closed doors: Promoting IRB transparency. J Law Med Ethics 2018;46(1),145-158. https://doi.org/10.1177\%2F1073110518766028

12. Emanuel EJ, Wendler D, Killen J, Grady C. What makes clinical research in developing countries ethical? The benchmarks of ethical research. J Infect Dis 2004;189(5):930937. https://doi.org/10.1086/381709

13. Coleman $\mathrm{CH}$, Bouësseau MC. How do we know that research ethics committees are really working? The neglected role of outcomes assessment in research ethics review. BMC Med Ethics 2008;9(6). https://doi.org/10.1186/1472-6939-9-6

14. Tsan MF. Measuring the quality and performance of institutional review boards. J Empir Res Hum Res Ethics 2019;14(3):187-189. https://doi. org/10.1177/1556264618804686

15. Kane EL, Gallo JJ. Perspectives of IRB chairs on the informed consent process. Am J Bioethics 2017;8(2):137-143. https://doi.org/10.1080/23294515.2016.125 3628

16. Klitzman R. How IRBs view and make decisions about consent forms. J Empir Res Hum Res Ethics 2013;8(1):8-9. https://doi.org/10.1525/jer.2013.8.1.8

17. Department of Health, South Africa. Ethics in Health Research: Principles, Structures and Processes. Pretoria: DoH, 2015.

18. World Medical Association. Declaration of Helsinki: Ethical principles for medical research involving human subjects. https://www.wma.net/policies-post/wmadeclaration-of-helsinki-ethical-principles-for-medical-research-involving-humansubjects/ (accessed 10 June 2018). 
19. Klitzman R. How good does the science have to be in proposals submitted to institutional review boards? An interview study of IRB personnel. Clin Trials 2013;10(5):761-766. https://doi.org/10.1177/1740774513500080

20. Benatar SR, Singer PA. Responsibilities in international research: A new look revisited. J Med Ethics 2010;36(4):194-197. https://doi.org/10.1136/ jme.2009.032672

21. Tindana P, Molyneux CS, Bull S, Parker M. Ethical issues in the export, storage and reuse of human biological samples in biomedical research: Perspectives of key stakeholders in Ghana and Kenya. BMC Med Ethics 2014;15(1):76. https://doi. org/10.1186/1472-6939-15-76

22. Lutge $E$, Slack C, Wassenaar D. Defining and negotiating the social value of research in public health facilities: Perceptions of stakeholders in a researchactive province of South Africa. Bioethics 2017;31(2):128-135. https://doi. org/10.1111/bioe. 12323

23. Barsdorf N, Millum J. The social value of health research and the worst off. Bioethics 2017;31(2):105-115. https://doi.org/10.1111/bioe.12320
24. Lairumbi GM, Parker M, Fitzpatrick R, English MC. Ethics in practice: The state of the debate on promoting the social value of global health research in resourcepoor settings, particularly Africa. BMC Med Ethics 2011;12(1):22. https://doi. org/0.1186/1472-6939-12-22

25. Wassenaar DR, Slack CM. How to learn to love your research ethics committee: Recommendations for psychologists. S Afr J Psych 2016;46(3):306-315. https:// doi.org/10.1177/0081246316654348

26. Angell EL, Dixon-Woods M. Do research ethics committees identify process errors in applications for ethical approval? J Med Ethics 2009;35(1):130-132. https://doi. org/10.1136/jme.2008.025940

Accepted 9 June 2019 